\title{
Characteristics of Ionospheric Scintillation in Chengdu, China
}

\author{
Song Zhang ${ }^{1}$, Hailong $\mathrm{Li}^{1}$, Jinghua $\mathrm{Li}^{2}$, Lin Meng ${ }^{1}$ \\ ${ }^{1}$ School of Electronic Science and Engineering, University of Electronic Science and Technology of China \\ ${ }^{2}$ National Astronomical Observatories, Chinese Academy of Sciences \\ Correspondence to: Hailong Li(hailong703@163.com)
}

\begin{abstract}
When wireless electromagnetic waves pass through the earth's atmosphere, they are affected by ionospheric irregularities, and their amplitude and phase will jitter rapidly in a short period of time, which is called ionospheric scintillation. With the human exploration of outer space and the demand for space communication, the study of ionospheric scintillation characteristics and its influence on electromagnetic communication has become increasingly important. This paper used the observation data received by GPS scintillation/TEC receivers in the Chengdu $\left(104.07^{\circ} \mathrm{N}, 30.67^{\circ} \mathrm{E}\right)$ area of China from January 2018 to September 2020, and a data processing program was developed for the received GPS/BDS/GAL. The satellite data at multiple frequency points were processed to extract key data such as S4, azimuth angle, and elevation angle, and then the annual changes in ionospheric scintillation in the Chengdu region and the characteristics of local time changes were statistically analysed. The results show that the frequency and intensity of ionospheric scintillation events have obvious half-year changes. The scintillation intensity and frequency in spring and autumn are higher and more frequent than those in summer and winter; and scintillation events mainly occur at night. but they also occur during the day, mostly in the afternoon; and their occurrence is related to the airspace and is further closely related to the elevation and azimuth angles of the observation point. The overall scintillation events from 2018 to 2020 were in a gradual downward trend. At the end of May 2018, a scintillation event with a longer duration occurred. Further analysis showed that the occurrence of scintillation events increased with the rapid changes in solar activity and the geomagnetic environment. There is a certain positive correlation between the changes.
\end{abstract}

Keywords amplitude; azimuth angle; ionosphere; scintillation event; solar activity; S4

\section{Introduction}

Affected by solar radiation and cosmic rays, the atmospheric molecules in the earth's atmosphere that are about $50 \mathrm{~km}$ to $251,000 \mathrm{~km}$ away from the ground are partially or completely ionized. This part of the atmosphere is called the ionosphere. Ionospheric scintillation refers to the phenomenon that the amplitude and phase of electromagnetic signals will jitter rapidly when wireless electromagnetic waves propagate through the ionosphere (Aarons, 1982; Kelley, 2009). It is generally believed that the cause of electromagnetic scintillation is the inhomogeneity of the ionospheric structure, such as the occasional E layer and the extended F layer. Ionospheric scintillation can cause distortion of the amplitude and phase of the electromagnetic signal 
and additional time delay, thereby affecting the accuracy and integrity of the signal, and even causing signal interruption. With the rapid development of modern communication and navigation technology, and the aerospace industry, the research on ionospheric scintillation has become increasingly important.

There are also many types of detection methods for the ionosphere, and the early and widely used methods are vertical gauge, digital altimeter and VHF radar observation. Since the 1980s, the development of navigation satellite technology has made the use of GPS signals for monitoring the most popular way (Basu et al., 1981; Van Dierendonck et al., 1993). Since the beginning of the new century, the requirements of space exploration technology and satellite-to-ground communication technology for communication reliability have continued to increase, requiring more accurate and detailed analysis of the impact of ionospheric scintillation on communication signals. In recent years, many scholars at home and abroad have analysed the regional ionospheric scintillation (Adewale et al., 2012; Akala et al., 2012; Amabayo et al., 2014; Xu et al., 2020; Deng et al., 2013; Huang et al., 2014; Balan et al., 2016; Zhang et al., 2019). They summarized many characteristics of ionospheric scintillation and accumulated a large amount of data, which greatly promoted the observation and research in the field of ionospheric scintillation. Shang et al. (2005) conducted a preliminary statistical analysis of the ionospheric scintillation event data in the L-band from July to December 2003 in Hainan, and found that ionospheric scintillation mainly occurred in the time period from sunset to midnight. It also shows that the increase in solar and geomagnetic activity is usually inversely proportional to the occurrence of ionospheric scintillation events. Li et al. (2005) studied a real-time monitoring system for ionospheric scintillation detection and display, which can monitor and analyse the amplitude and phase scintillation index of GPS signals in real time, so as to automatically receive and store the received data such as ionospheric amplitude and phase, which further promoted the research of automated real-time monitoring of ionospheric scintillation. Deng et al. (2007) analysed the total electron content (TEC) of the GPS ionosphere in Wuhan and the measurement data of the electric scintillation detector,

50 and found that during a magnetic storm, the ionospheric TEC in Wuhan and its surroundings mainly responded to the positive storm phase. Zhang et al. (2019) used the observational data of ionospheric scintillation in Shenzhen of the Meridian Project and through comparative analysis, found that the scintillation in the Shenzhen area mainly occurred between 19:00 and 2:00 in the local time. The occurrence rate of ionospheric scintillation and the loss of signal lock are good time correlation. Many scholars have analysed the characteristics of ionospheric scintillation from different aspects, laying a solid foundation for the study of the regional occurrence characteristics of ionospheric scintillation, and it is of great significance for the further development of the research on ionospheric physical characteristics.

However, combined with existing research findings, the research on the characteristics of ionospheric scintillation regions in China is mainly concentrated in South China and East China. Southwest China, which is also in the hump region of the magnetic equatorial anomaly region (Aarons, 1993), is yet to be analysed in terms of the characteristics of ionospheric 60 scintillation. It is relatively rare, and related data are relatively lacking. Southwest China is also an area with a comparatively complex meteorological environment in the country, which has important practical significance for the ionospheric exploration research in this region. This paper used ionospheric scintillation data from January 2018 to September 2020 in Chengdu, China, 
for nearly three consecutive years, to study and analyse the temporal and spatial characteristics of ionospheric scintillation events in Chengdu.

The ionospheric scintillation data used in this article came from the observation data received by the GPS scintillation/TEC receiver in the Chengdu $\left(104.07^{\circ} \mathrm{N}, 30.67^{\circ} \mathrm{E}\right)$ area of the University of Electronic Science and Technology of China from January 2018 to December 2020. The ionospheric scintillation receiver uses ultra-low phase noise ovencontrolled crystal oscillator (OCXO) as the frequency source of the receiver. Records of the signal strength, pseudo-range and phase observations of GPS and BDS visible satellites at a sampling frequency of $50 \mathrm{~Hz}$, at the same time, choke antennas were used to reduce the impact of multipath effects on the observation data. The satellite signals received by the ionospheric scintillation receiver came from multiple satellite systems. The GPS has L1/L2/L5 frequency bands; the BDS signals have B1/B2 frequency bands; and the Galileo signals have E1/E5 frequency bands, and each satellite system can track up to 14 satellites. The tracking sensitivity is $32 \mathrm{dBHz}$, and the amplitude scintillation index update frequency is once per minute. At

75 the same time, the device can also monitor the phase scintillation index and ionospheric TEC data. This article only analysed the ionospheric amplitude scintillation index, mainly using BDS data, and also used GPS and Galileo signal data to reduce statistical errors.

Ionospheric amplitude scintillation is the amplitude change caused by the electromagnetic wave signal passing through the ionosphere. It is generally measured by the ionospheric amplitude scintillation index, S4. In Wang et al. (2020), S4 is defined as the normalized difference between the scintillation amplitude and the signal intensity of the system noise per minute. The S4 formula is given as follows.

$$
S 4=\sqrt{\frac{\left\langle S I^{\prime} 2\right\rangle-\left\langle S I^{\prime}\right\rangle^{2}}{\left\langle S I^{\prime}\right\rangle^{2}}-\frac{100}{S / N_{0}}\left(1+\frac{500}{19 S / N_{0}}\right)}
$$

Among them, $S I^{\prime}$ is the one-minute mean value of the signal strength $S I$ after filtering, and $S / N_{0}$ is the mean value of the signal-to-noise ratio output by the system. To study the occurrence of ionospheric scintillation under different intensities, we referred to the S4 intensity reference (Guo et al., 2017), then, combined with the scintillation data in the Chengdu area, and divided the ionospheric scintillation into four intensity levels: $\mathrm{S} 4<0.2$ means no scintillation, $0.2<\mathrm{S} 4<0.4$ means weak scintillation, $0.4<\mathrm{S} 4<0.6$ means medium scintillation, and $\mathrm{S} 4>0.6$ means strong scintillation. At the same time, because the satellite is easily affected by the multipath effect when the elevation angle is low, we intercepted the data with the elevation angle above $20^{\circ}$ for processing to reduce the error caused by the multipath effect on the ionospheric scintillation index.

The incidence of ionospheric scintillation is defined as follows:

$$
\text { Occurrence rate }=\frac{\text { number }(S 4 \geq \text { threshold })}{\text { total }(S 4 \geq 0.2)} \times 100
$$




$$
\text { Occurrence rate }=\frac{\text { number }(S 4 \geq \text { threshold })}{\text { total }(S 4 \geq 0.2)} \times 100
$$

Due to the complex conditions of meteorological addresses in the Sichuan Basin and frequent earthquakes, some data were severely distorted due to noise, and some data were not received effectively due to system software upgrades and hardware loss. Because this part of the data seriously affects the analysis results, we considered removing this part of the data. Further, because the received data came from multiple frequency bands of multiple satellite systems, although part of the data is lost at the same time, there are still some valid observation data. Comparing and calculating adjacent data will basically not affect the analysis results.

To further improve the accuracy of data analysis, referring to the work of Huang et al. (2014), a scintillation event with an S4 index of not less than 0.2 and lasting at least 5 minutes is defined as a scintillation event, and the scintillation event is in the frequency bands of the two satellite systems. The scintillation event can be observed before being counted. This helps to eliminate short-term signal amplitude fluctuations caused by non-ionospheric factors, and ultimately, still count enough scintillation events for analysis.

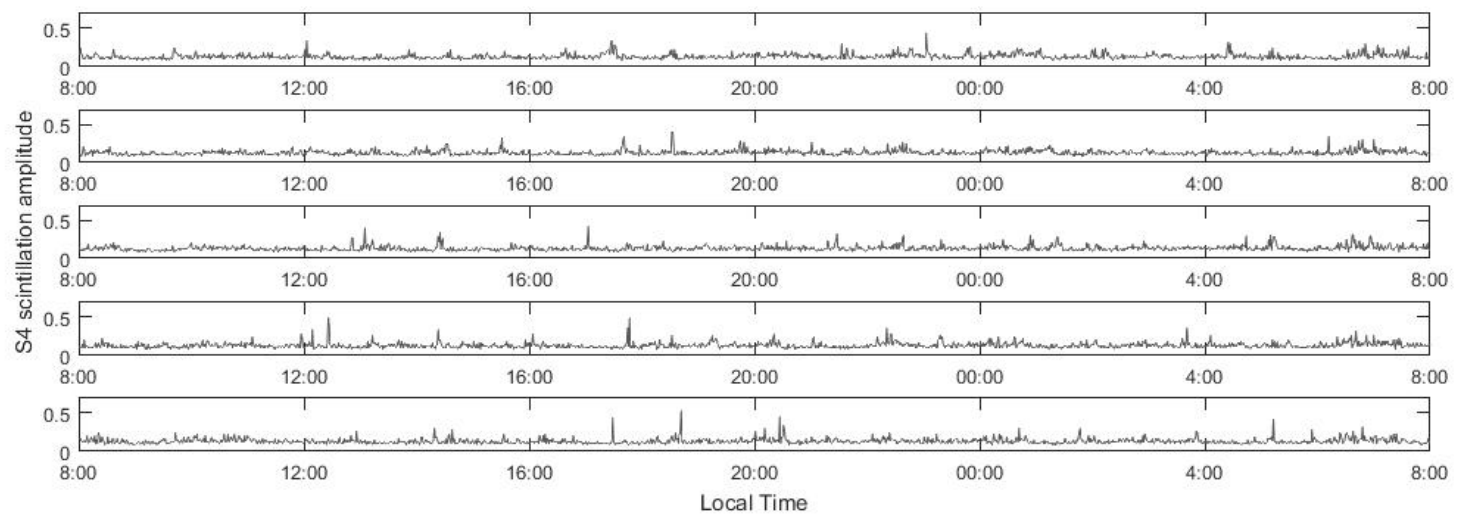

Fig. 1 Changes in the ionospheric amplitude scintillation on July 1-5, 2019, with local time

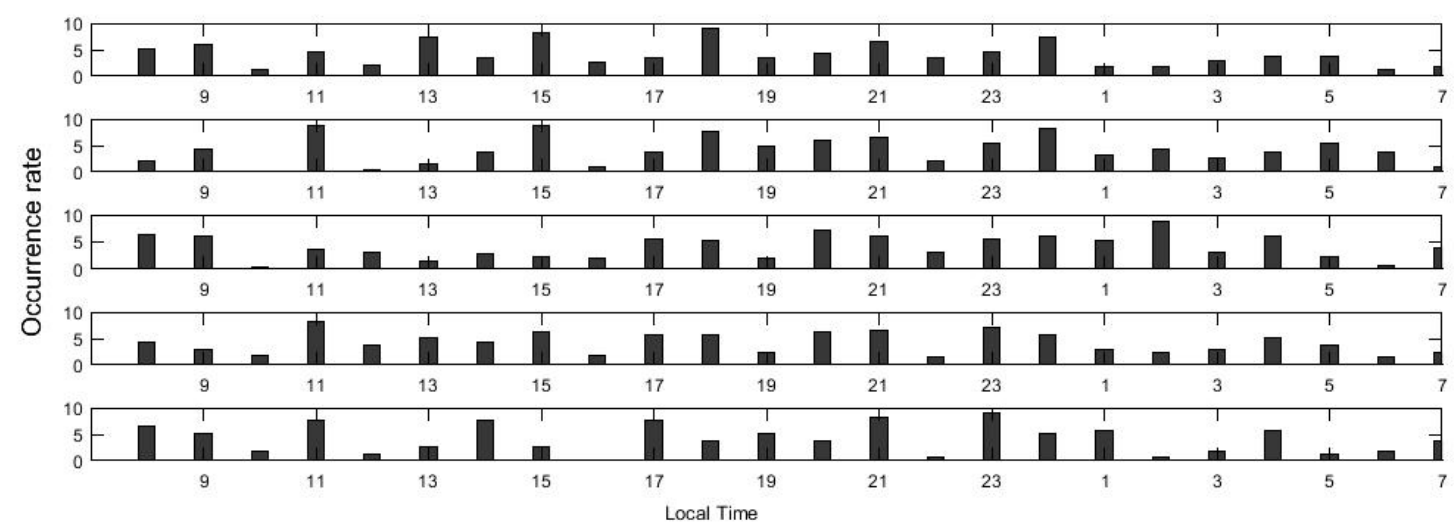

Fig. 2 Changes in the incidence of different intensities on July 1-5, 2019, with local time 


\section{Processing results and analysis}

\subsection{Temporal and seasonal changes of ionospheric scintillation}

The local time variation of the ionospheric amplitude scintillation intensity for a total of 5 days from July 1-5, 2019 is shown in Figure 1. To analyse the daily distribution of different intensities of the ionospheric amplitude scintillation, the incidence of ionospheric amplitudes of different intensities each day is plotted in Figure 2. The incidence rate is defined as the ratio of the number of abnormal occurrences of ionospheric scintillation per day to the total number of ionospheric scintillations received. It can be clearly analysed from the figure that the 5-day scintillation mainly occurred between 17:00 and 1:00 local time. In addition, denser scintillation events occurred from 4:00 to 8:00, and sporadic strong scintillation events also occurred in the afternoon, but there are also differences. The strong scintillation events in the next 3 days are higher than those in the previous 2 days, and the occurrence of scintillation events in the next 3 days is obviously shifted back in time. From the perspective of the evolution process of ionospheric irregularities that cause ionospheric scintillation, the incidence of such ionospheric scintillation is related to the occurrence, development and attenuation of ionospheric irregularities (Zhang et al., 2019). From the point of view of ionospheric scintillation effects, the influence of strong ionospheric scintillation events is greater than that of weak ionospheric scintillation events. Therefore, the understanding of changes in ionospheric scintillation intensity distribution is meaningful in theory and application.
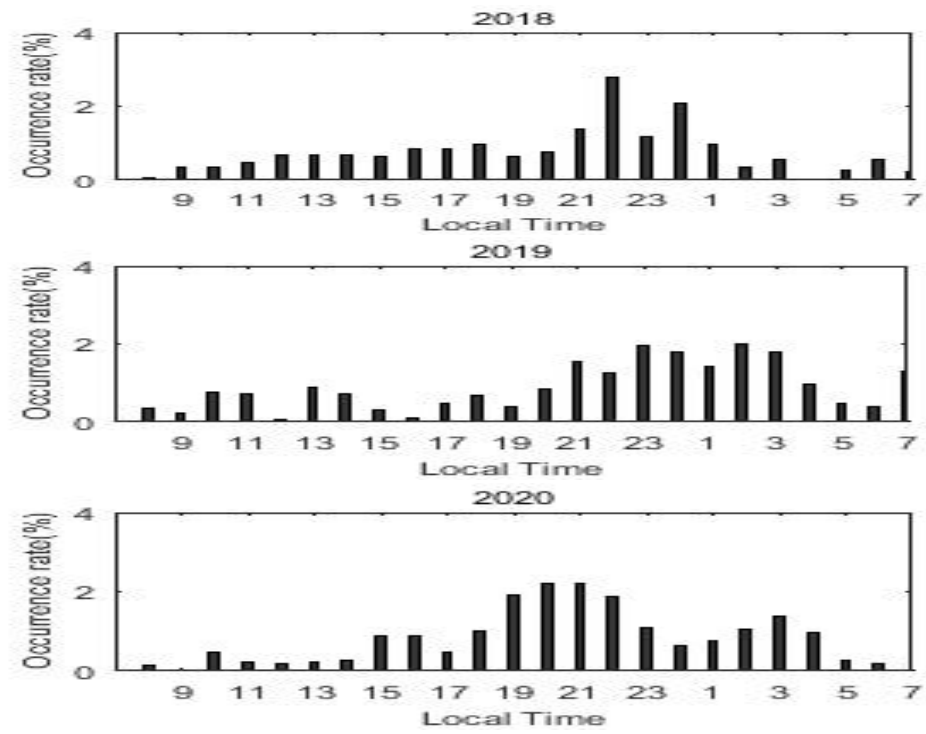

Fig. 3 Changes in the occurrence rate of ionospheric scintillation events from 2018 to 2020, with local time

Figure 3 shows the statistical law of the local time variation of the occurrence rate of scintillation with different intensity amplitudes in each year from 2018 to 2020. It is obvious from the figure that the ionospheric scintillation events mainly occur at around 17:00 to 3:00 local time, and there is also a higher incidence of scintillation events in the afternoon. This is basically consistent with the scintillation event rule we analysed for a single day in July, which is related to the solar radiation of different 
intensities that the ionosphere receives during the diurnal variation. At the same time, the scintillation events of different intensities basically meet the above rules, but generally, the incidence of weak scintillation is greater than that of moderate scintillation and that of strong scintillation. This is also in line with the relevant statistical studies of other scholars (Xiong B et al., 2007; Zhang et al., 2019; Zuo et al., 2014), and the stronger the ionospheric scintillation event, the greater the impact on human activities.
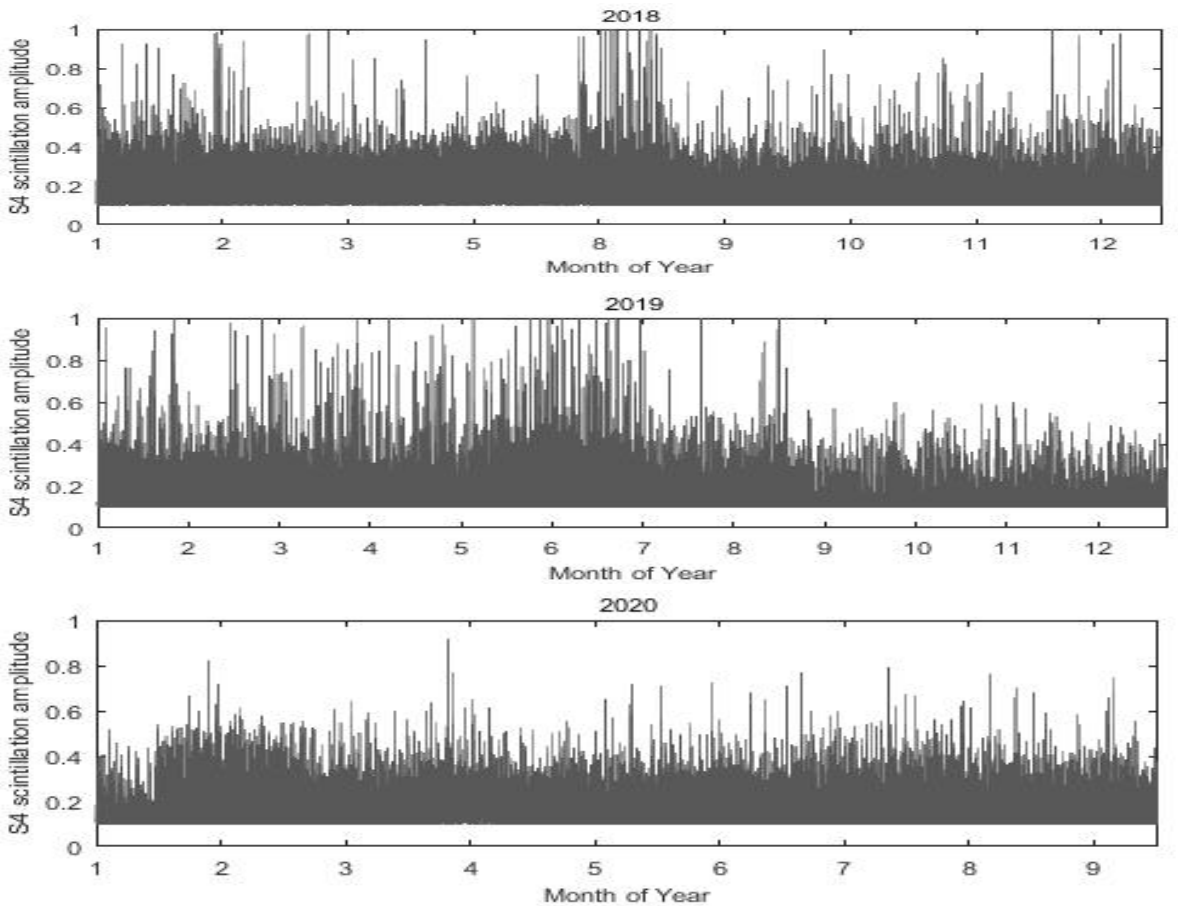

Fig. 4 Seasonal changes in the scintillation amplitude from 2019 to 2020
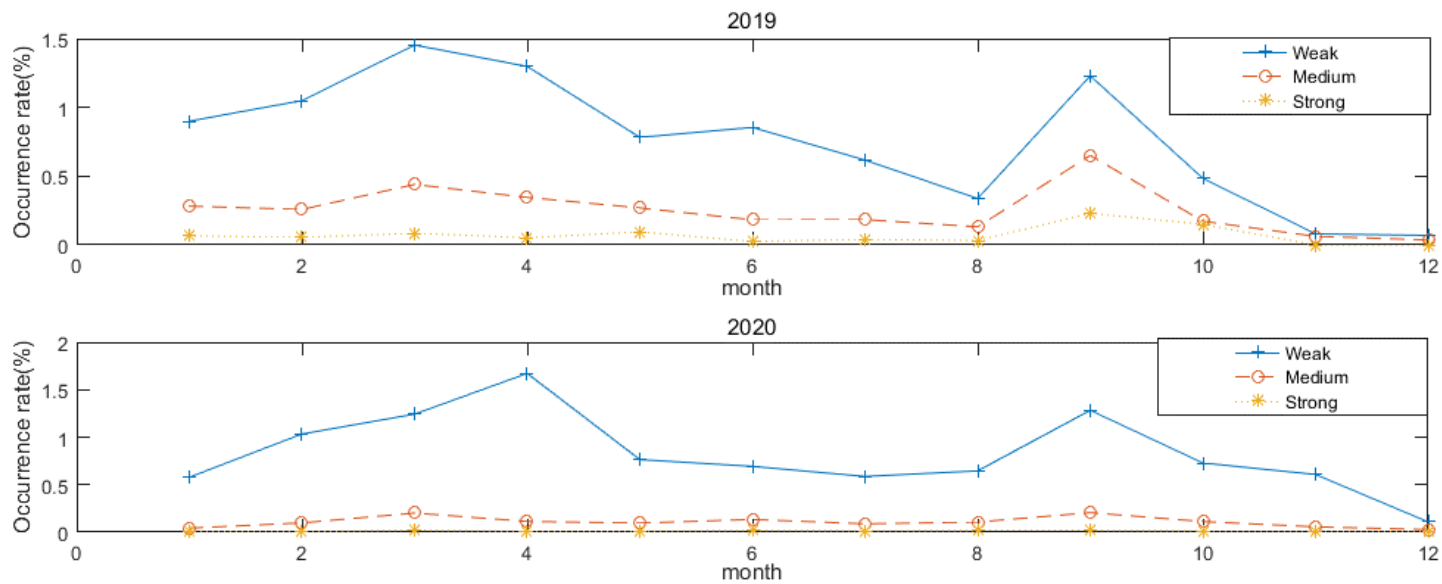

Fig. 5 Seasonal changes in the occurrence of scintillation events from 2018 to 2020 
The statistical results of scintillation events with different intensity amplitudes in each year from 2018 to 2020 are shown in Figure 4, and the different occurrence rates of the ionosphere with daily changes in each year are shown in Figure 5. It shows that the incidence and intensity of scintillation events have obvious seasonal changes. The scintillation events of each year mainly occur in spring and autumn equinox (March-April and September-October). In May 2018, a long-lasting ionospheric scintillation event occurred. Analysis of the ionospheric scintillation data during this time period revealed that the scintillation events occurred mainly at night and the events were mainly strong scintillation events. According to the results of previous studies, the increase in the intensity of solar activity will cause the electron density in the ionospheric structure to fluctuate randomly (Fejer et al., 1979; Rastogi et al., 1980), and the occurrence rate of ionospheric scintillation events. Of course, it cannot be ruled out that the statistical results of scintillation events are abnormal due to different elevation angle distributions or the influence of the geomagnetic environment. Further analysis was then conducted. The figure also shows that the incidence of scintillation events is decreasing year by year. In 2018, there are basically high scintillation events. By 2020, except for the high scintillation events in January and September, the scintillation rates of the remaining months are relatively low. The year-to-year change in the incidence of this scintillation is related to the periodicity of solar activity (Guo et al., 2017).

The scintillation characteristics are closely related to the generation and evolution principle of ionospheric irregularities. Ionospheric irregularities generally depend on the extended F layer and the occasional E layer. In areas with low geomagnetic latitudes, the wind speed of the equivalent wind field at night and noon is higher than other times of the day, which affects the distribution of electron density and changes in geomagnetic activity. The structure of electric field and wind field, and so the diurnal and seasonal changes of ionospheric scintillation are also related to the lower geomagnetic latitude and equivalent wind field changes in Chengdu.

\subsection{Spatial distribution characteristics of ionospheric scintillation}

Through the analysis of all scintillation signal data received from 2018 to 2020, the probability of occurrence of ionospheric scintillation events with the azimuth angle is shown in Figure 6 . The range of azimuth angle is $0^{\circ}-360^{\circ}$, and the range of elevation angle is $20^{\circ}-90^{\circ}$. The figure shows that the amplitude scintillation has obvious azimuth distribution characteristics. Although there are certain scintillation events in each azimuth angle range, the ionospheric scintillation events mainly occur between the azimuth angle range of $150^{\circ}-210^{\circ}$ and the amplitude of different intensity. There are some differences in scintillation. From the perspective of medium intensity scintillation and strong scintillation events, they are mainly concentrated in the azimuth angle of about $150^{\circ}-210^{\circ}$, and there is a higher incidence at about $60^{\circ}$, especially in 2018. The weak scintillation events as a whole also conform to the distribution law of strong scintillation events, but there is also a certain proportion of scintillation events at other angles and azimuths. Other scholars have also given similar spatial distribution of ionospheric scintillation in East Asia (Abadi et al., 2014; Kelley, 2009; Tran et al., 2017). In addition, this spatial distribution is explained based on the characteristics of the ionospheric heterogeneous structure. Chengdu is also located at the northern 
https://doi.org/10.5194/angeo-2021-13

Preprint. Discussion started: 19 February 2021

(c) Author(s) 2021. CC BY 4.0 License.

(c) (i)

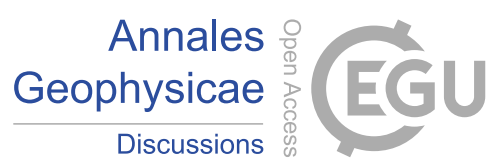

edge of the hump region of the magnetic equatorial anomaly area. The ionospheric heterogeneity is generally accompanied by the arrangement of magnetic lines. The comparison of the geomagnetic declination in Chengdu Low, when most of the observation satellites are located in the southern direction and the elevation angle is relatively low, the radio electromagnetic waves will take longer to pass through irregularities, and the delay effect of the electromagnetic waves will gradually accumulate and amplify, thereby receiving more ionospheric scintillation event.
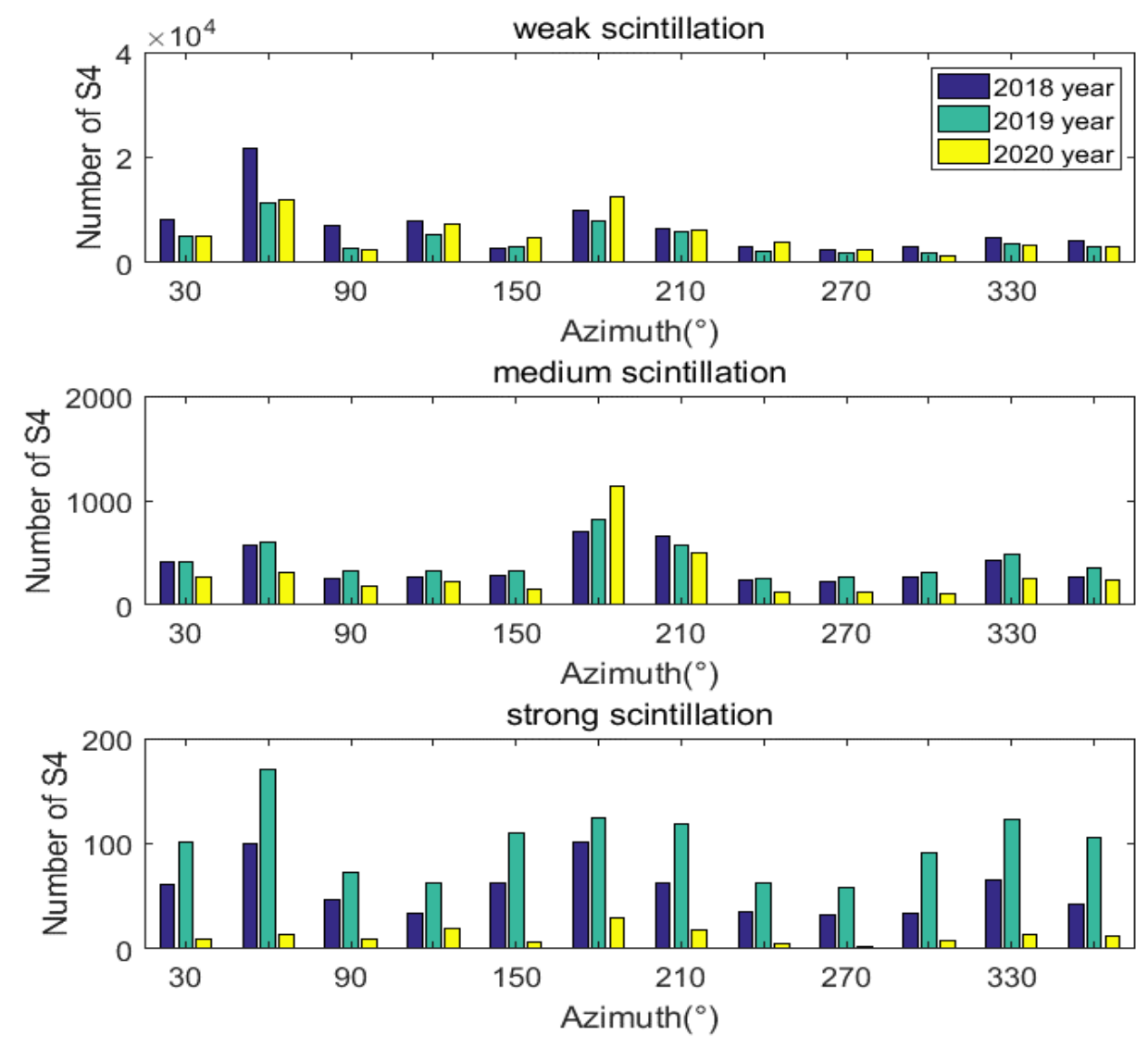

Fig. 6 Changes in the incidence of scintillation events with azimuth from 2018 to 2020

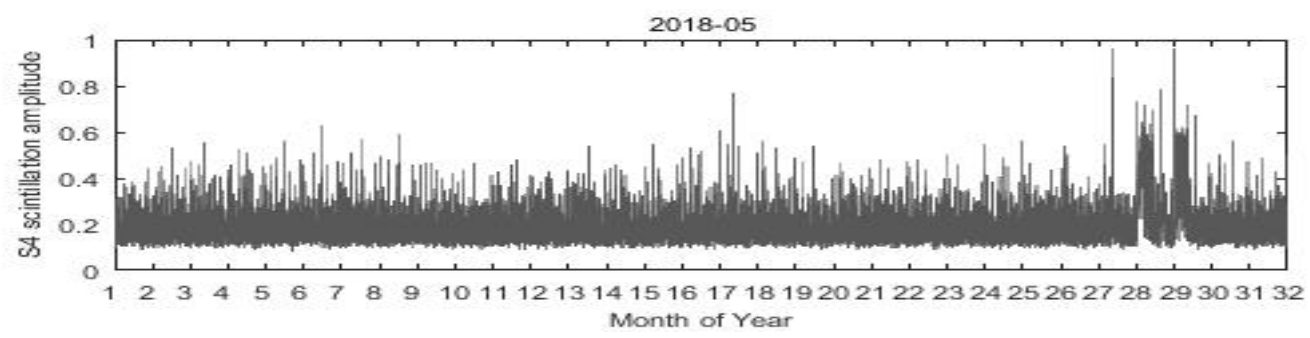

Fig. 7 Changes in the location of scintillation events in May 2018, with local time 


\subsection{The relationship between ionospheric scintillation and solar activity}

In recent years, in low and middle latitudes, different scholars have studied the correlation between ionospheric scintillation incidence and solar activity based on ionospheric scintillation data in different places. The results show that ionospheric scintillation events occur in low and middle latitudes. There is a clear dependence on the change of solar activity rate and intensity (Xu et al., 2020; Shi et al., 2011; Zhang et al., 2010). A long-term ionospheric scintillation event was observed from about May 27-30, 2018 (Figures 7 and 8) showing that scintillation events with moderate intensity or above are the main ones, which roughly occurred at around 8 to 20 o'clock in the local time. The observations of some parameters of solar activity from May 27 to 30 UTC are shown in Figure 9 noting that the electron flux and X-ray flux parameters have appeared from May 28 to 29 UTC. For the anomalies, there is a strong correlation between ionospheric scintillation anomalies and changes in solar activity intensity. Luo et al. (2013) concluded that the linear growth rate of generalized R-T instability is positively correlated with the level of solar activity through theoretical calculations.

In studying ionospheric scintillation events, solar activity is also one of the main factors that must be considered. However, Zhang et al. (2019) found that the incidence of ionospheric scintillation and solar activity are not necessarily linear. In addition, the peaks of the daily rate of change of the ionosphere at night and in the afternoon are also related to the different levels of ionospheric ionization caused by different levels of ionization of air molecules of different heights and compositions during the day.

\section{Summary}

Using the GPS/BDS/GAL ionospheric scintillation receiver observation data in the Chengdu area from January 2018 to September 2020 in the past 3 years, a combined analysis of the amplitude scintillation values of different frequency points was carried out, and the correlation of meteorological satellite statistics was used. Solar activity data were also analysed. The results are as follows:

The activity of ionospheric scintillation events monitored in the Chengdu area shows a seasonal distribution pattern of strong spring and autumn equinox (March-April and September-October) and weak winter and summer (November-February and May-August). They occur frequently from 17:00 to 3:00 in the local time, and a small number of strong scintillation events occur after noon, and the frequency of scintillation with different intensities varies with the local time.

In terms of the distribution of scintillation regions, ionospheric scintillation events mainly occurred at an azimuth angle of $120^{\circ}-200^{\circ}$, strong scintillation events concentrated at an azimuth angle of $160^{\circ}$, and a relatively concentrated scintillation event also appeared at an azimuth angle of $40^{\circ}$. A considerable number of weak scintillation events occur throughout the year, and the overall distribution also meets the law of occurrence of strong scintillation events, but overall, the occurrence of strong scintillation events is less than that of weak scintillation events. 
https://doi.org/10.5194/angeo-2021-13

Preprint. Discussion started: 19 February 2021

(c) Author(s) 2021. CC BY 4.0 License.
Annales

Geophysicae

Discussions

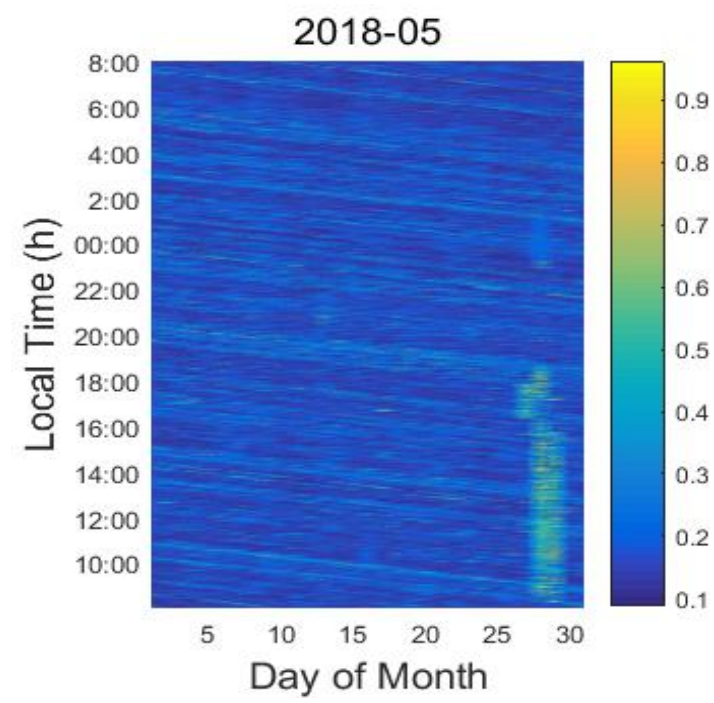

Fig. 8 Changes in scintillation events in May 2018 with local time and monthly accumulation days
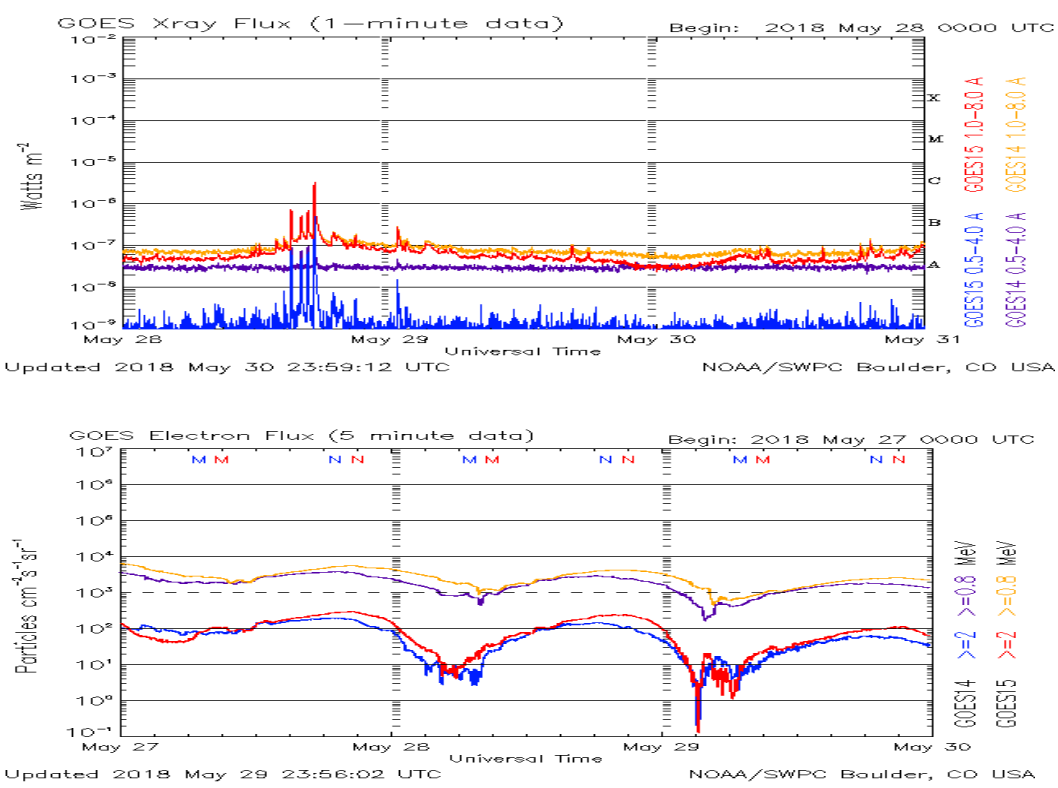

Fig. 9 Changes in electron flux parameters observed at the end of May 2018

\section{Acknowledgments}

This work is supported by the National Natural Science Foundation of China (No. 61671116, 61771096, 11905026). This study is also supported by the National Key Laboratory of Electromagnetic Environment, China Research Institute of Radiowave Propagation. 


\section{References}

Aarons, J.: Global morphology of ionospheric scintillation, Proc. IEEE, 70, 360-378, 1982.

Aarons, J.: The longitudinal morphology of equatorial F-layer irregularities relevant to their occurrence, Space Sci Rev. 63, 209-243, 1993.

Abadi, P, Saito, S, and Srigutomo, W.: Low-latitude Scintillation Occurrences around the Equatorial Anomaly Crest over Indonesia, Annales Geophysicae. 32(1), 7-17, 2014.

Akala, A.O, and Doherty, P.H.: Statistical Distribution of GPS Amplitude Scintillations, Journal of Atmospheric and Solarterrestrial Physics. 74, 199-211, 2012.

Amabayo, Emirant Bertillas, Edward, Jurua, Cilliers, Pierre J, and Habarulema, John Bosco.: Climatology of Ionospheric Scintillations and TEC Trend over the Ugandan Region. Advances in Space Research. 53(5),734-43, 2014.

Basu, S.: Equatorial scintillations-A review. J Atmos Terrestrial Phys. 43: 473-489, 1981.

Deng, Baichang, Huang, Jiang, Liu, Weifeng, Xu, Jie, and Huang, Lingfeng.: GPS Scintillation and TEC Depletion near the Northern Crest of Equatorial Anomaly over South China. Advances in Space Research 51(3), 356-65, 2013.

Fejer, B.G, Farley, D.T, Woodman, R.F, and Calderon, C.: Dependence of Equatorial F Region Vertical Drifts on Season and Solar Cycle. Journal of Geophysical Research: Space Physics. 84( A10), 5792-796, 1979.

Guo, Kai, Zhao, Yan, Liu, Yang, Wang, Jinling, Zhang, Chunxi, and Zhu, Yanbo.: Study of Ionospheric Scintillation Characteristics in Australia with GNSS during 2011-2015. Advances in Space Research. 59(12), 2909-922, 2017.

Huang, Linfeng, Wang, Jinsong, Jiang, Yong, Chen, Zhou, and Zhao, Kai.: A Study of GPS Ionospheric Scintillations Observed at Shenzhen. Advances in Space Research. 54(11), 2208-2217, 2014.

Jerez, Gabriel Oliveira, Alves, Daniele Barroca Marra, and Tachibana, Vilma Mayumi.: Multivariate Analysis of Combined GPS/GLONASS Point Positioning Performance in Brazilian Regions under Different Ionospheric Conditions. Journal of Atmospheric and Solar-terrestrial Physics. 187, 1-9, 2019.

Jiang, ChunHua, Wei, LeHui, Yang, GuoBin, Zhou, Chen, and Zhao, ZhengYu.: Numerical simulation of the propagation of electromagnetic waves in ionospheric irregularities. Earth and Planetary Physics, 4(6), 565-570, 2020.

Kelley M C. The Earth's Ionosphere: Plasma Physics and Electrodynamics. Burlington: Academic Press, 2009.

Balan, Nanan, Liu, LiBo, and Le, HuiJun.: A brief review of equatorial ionization anomaly and ionospheric irregularities. Earth and Planetary Physics, 2(4), 257-275, 2018.

Rastogi, R G.: Seasonal and solar cycle variations of equatorial spread-F in the American zone. J Atmos Terr Phys. 42: 593597, 1980.

Seba, Ephrem Beshir, and Gogie, Tsegaye Kassa.: Characterization of Ionospheric Scintillation at a Geomagnetic Equatorial Region Station. Advances in Space Research. 56(10), 2057-063, 2015.

250 Shi, J K, Wang, G J, Reinisch B W, et al.: Relationship between strong range spread F and ionospheric scintillations observed in Hainan from 2003 to 2007. J Geophys Res., 116: A08306, 2011. 
https://doi.org/10.5194/angeo-2021-13

Preprint. Discussion started: 19 February 2021

(c) Author(s) 2021. CC BY 4.0 License.

(c) (1)

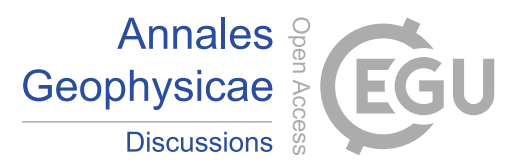

Tran, T L, Le, H M, Amory-Mazaudier C, et al.: Climatology of ionospheric scintillation over the Vietnam low-latitude region for the period 2006-2014. Adv Space Res., 60: 1657-1669, 2017.

Wang, Cheng, Fan, Lei, Wang, Zhipeng, and Shi, Chuang.: Assessment of Global Ionospheric Maps over Continental Areas Using Precise Point Positioning Technique. Journal of Spatial Science, 65(1), 25-39, 2020.

Van Dierendonck, A. J., Hua, Q., and Klobuchar, J.: Ionospheric scintillation monitoring using commercial single frequency C/A code receivers, Proceedings of ION GPS 93, Salt Lake City, UT,22-24 September, 1333-1342, 1993.

$\mathrm{Xu}$, Cheng J, Xu J S.: Statistical feature of TEC fluctuations and phase scintillations in the low latitude ionosphere of China. Chinese J. Geophys. (in Chinese), 63(1): 19-30, doi: 6038/cjg2019M0064, 2020.

260 Yeh, K. C. and Liu, C. H.: Radio wave scintillations in the iono-sphere, Proc. IEEE, 70 (4), 324-360, 1982.

Zhang D H, Xiao Z, Feng M, et al.: Temporal dependence of GPS cycle slip related to ionospheric irregularities over China low-latitude region. Space Weather, 8: S04D08, 2010.

Zhang M, Zhang D H, Hao Y Q, et al.: A statistical study on L-band ionospheric amplitude scintillation in Shenzhen during 2011-2016 (in Chinese). Sci Sin Tech, 49: 1570-1582, doi: 10.1360/SST-2019-0093, 2019. 